\title{
Термоэдс и термомагнитные свойства твердых растворов $\mathrm{Er}_{x} \mathrm{Sn}_{1-x} \mathrm{Se}$
}

\author{
(С) Дж.И. Гусейнов ${ }^{1}$, М.И. Мургузов ${ }^{1}$, Ш.С. Исмаилов ${ }^{1}$, Р.Ф. Мамедова ${ }^{1}$, Э.М. Годжаев ${ }^{2}$ \\ ${ }^{1}$ Азербайджанский государственный педагогический университет, \\ AZ-1000 Баку, Азербайджан \\ ${ }^{2}$ Азербайджанский технический университет, \\ AZ-1073 Баку, Азербайджан \\ E-mail: cih_58@mail.ru
}

(Получена 18 мая 2016 г. Принята к печати 20 мая 2016 г.)

В системе $\operatorname{Er}_{x} \mathrm{Sn}_{1-x} \mathrm{Se}$ наблюдаются сильное отклонение от линейности температурной зависимости дифференциальной термоэдс при температурах ниже комнатной и изменение знака коэффициента термомагнитных эффектов. Выявлено, что при неравновесном состоянии в исследуемых составах изменение от линейности термоэдс связано в основном с увлечением носителей заряда фононами $\alpha_{\mathrm{ph}}$. Оценены статистические силы электронного увлечения $A_{\mathrm{ph}}(\varepsilon)$.

DOI: 10.21883/FTP.2017.02.44097.7618

\section{1. Введение}

Изучению физических свойств полупроводниковых термоэлектрических материалов типа SnSe и его аналогов посвящено много исследований [1-3]. Эта группа кристаллов, особенно $\mathrm{SnSe}$, привлекла к себе внимание тем, что, с одной стороны, они являются термоэлектрическими материалами, а с другой - благодаря широкой запрещенной зоне $(0.9$ эВ) интересны с точки зрения оптических свойства, т.е. обладают промежуточными характеристиками термоэлектрических и оптических материалов [4,5]. SnSe кристаллизируется в структуру типа деформированного $\mathrm{NaCl}$ и имеет сложную ковалентноионную химическую связь [6]. Особенностью $\mathrm{SnSe}$ является наличие в значительной степени ассоциации дефектов $[3,4]$ и вакансий в обеих подрешетках. Высокая концентрация этих дефектов, особенно вакансий олова $\left(\sim 10^{17} \mathrm{~cm}^{-3}\right)$, обусловливает положительный тип проводимости в $\mathrm{SnSe}$. Частичное замещение олова примесями редкоземельных металлов, например эрбием (Er), приводит к возникновению ряда физических особенностей в $\mathrm{SnSe}$, обусловленных сложной природой дефектообразования и взаимодействием дефектов $[6,7]$.

Ранее нами были исследованы тепло- и электрофизические свойства этих систем [6,7] и выявлено, что в исследуемых твердых растворах $\mathrm{Er}_{x} \mathrm{Sn}_{1-x} \mathrm{Se}$ имеет место смена знака носителей заряда в зависимости от количества замещающего эрбия $(x \geq 0.005$ ат\%), в образцах $\mathrm{SnSe}$ происходит частичная самокомпенсация, т.е. $\operatorname{Er}_{x} \mathrm{Sn}_{1-x} \mathrm{Se}$ является компенсированным полупроводниковым материалом со сложным механизмом компенсации [8]. С ростом содержания примеси $\mathrm{Er}$ заметно уменьшение общей концентрации и подвижности носителей заряда. Однако до конца не ясна природа механизма рассеяния носителей заряда и тепла, и имеются смутные представления об электронно-фононных и фонон-фононных взаимодействиях, которые влияют на кинетические параметры. В настоящей работе, яв- ляющейся продолжением [6,7], приводятся результаты исследований термоэдс и термомагнитных свойств этих материалов.

\section{2. Экспериментальная часть}

Методы получения, физико-химический анализ, а также исследование электропроводности и эффекта Холла образцов подробно описаны в работах [6,7]. Нумерация (1-8) синтезированных образцов отвечала составам: $1-x=0.00,2-0.0001,3-0.0025,4-0.005$, $5-0.01,6-0.015,7-0.025,8-0.050$. Термоэдс и термомагнитные коэффициенты измеряли стационарным методом компенсации. Магнитное поле напряженностью $H=11000$ Э направлено вдоль [001], а постоянный электрический ток вдоль [100]. В интервале температур $T=77-700 \mathrm{~K}$ были исследованы термоэдс $(\alpha)$, коэффициенты поперечного $\left(E_{y}\right)$ и продольного $\left(\Delta \alpha / \alpha_{0}\right)$ эффекта Нернста-Эттингсгаузена $(\mathrm{H}-Э)$. Погрешность измерений термоэдс составляла $4.6 \%$, а поперечных и продольных безразмерных коэффициентов Н-Э не превышала $\sim 7.1 \%$.

\section{3. Обсуждение результатов}

Образцы 1-3 (включая исходный $p$-SnSe) имеют дырочную проводимость, концентрация $5.7 \cdot 10^{18}$, $3.9 \cdot 10^{16} \mathrm{~cm}^{-3}$ в образцах 1 и 3 соответственно. Образцы с последующими номерами 4-8 имеют электронную проводимость, и концентрация носителей заряда с увеличением содержания эрбия в образцах возрастает от $1.4 \cdot 10^{16} \mathrm{~cm}^{-3}$ до $5.4 \cdot 10^{17} \mathrm{~cm}^{-3}$ при $T=90 \mathrm{~K}$. На рис. 1 представлены кривые температурных зависимости термоэдс $\left(\alpha_{\text {tot }}\right)$ образцов. Величина $\alpha_{\text {tot }}$ с ростом температуры до $400 \mathrm{~K}$ изменяется нелинейно, а при $T>440 \mathrm{~K}$ плавно уменьшается: причем в образцах с дырочной проводимостью уменьшение $\alpha_{\text {tot }}$ намного резче, чем с 


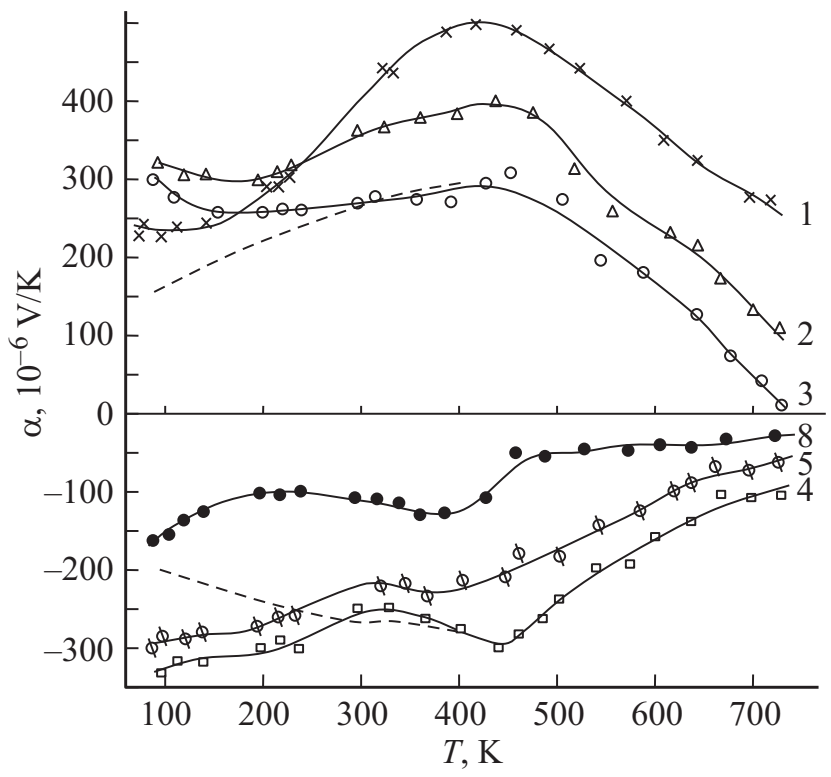

Рис. 1. Зависимости термоэдс $\alpha$ от температуры в твердых растворах $\mathrm{Er}_{x} \mathrm{Sn}_{1-x} \mathrm{Se}$. Образцы: $1-x=0,2-0.001$, $3-0.0025,4-0.005,5-0.01,8-0.05$. Сплошные кривые - эксперимент $\left(\alpha_{\mathrm{tot}}\right)$, штриховые - расчет $\left(\alpha_{T}\right)$ для образцов 3,4 .

проводимостью $n$-типа. В то же время при одной и той же температуре в образце с увеличением содержания $\mathrm{Er}$ значение $\alpha_{\text {tot }}$ по абсолютной величине заметно уменьшается. Для сравнения и выяснения анализа полученных экспериментальных данных по $\alpha_{\text {tot }}(T)$ проведены теоретические расчеты термоэдс для образцов 3 и 4 по формуле $[9,10]$

$$
\alpha_{T}= \pm \frac{k_{0}}{e}\left[\frac{(r+2) F_{r+1}(\bar{\mu})}{(r+1) F_{r}(\bar{\mu})}-(\bar{\mu})\right]
$$

для параболической изотропной зоны (при $m^{*}=$ const), результаты приведены на рис. 1 для образцов 3 и 4 (штриховые кривые). Из рис. 1. видно, что экспериментальные значения $\alpha_{\text {tot }}(T)$ значительно превышают теоретические значения $\alpha_{T}(T)$. Оценка, проведенная при $96 \mathrm{~K}$, показывает, что для образца $3 p$-типа проводимости $\alpha_{\text {tot }} / \alpha_{T}=0.433=43 \%$, для образца $4 n$-типа проводимости превышает $\sim 38 \%$. Попытка объяснить разность между $\alpha_{\text {tot }}(T)$ и $\alpha_{T}(T)$ и отступление от линейности в рамках простой теории компенсации легирующего действия примеси одиночными вакансиями не увенчалась успехом. Согласно данным ранее опубликованных нами работ [7], намного лучшие результаты получаются в том случае, если предположить, что компенсация легирующего действия примеси $\mathrm{Er}$ осуществляется не только вакансиями, но и путем образовании комплексов [8]. В обычных условиях примесь эрбия в $\mathrm{SnSe}$ образует акцепторы. Однако, поскольку в $\mathrm{SnSe}$ олово с халькогеном двухвалентно, его частичное замещение трехвалентным эрбием, по-видимому, приводит к появлению мелких донорных уровней, обусловленных участием вакансии олова: $2 \mathrm{Er}_{\mathrm{Se}}^{0} \rightarrow \mathrm{Er}_{\mathrm{Se}}^{-}+\left(\mathrm{Er}_{i} V_{\mathrm{Sn}}\right)^{+}$. Согласно данным работы [7], для компенсированных образцов $p$ - и $n$-типа проводимости характерно незначительное уменьшение коэффицента Холла (за исключением образцов 2, 3, где наблюдается $p$-тип проводимости) с ростом температуры. При низких температурах (до $200 \mathrm{~K}$ ) это падение можно связать с ионизацией донорных центров с концентрацией $N_{D}=10^{16} \mathrm{~cm}^{-3}$. Из температурной зависимости электропроводности определены энергии активации уровней, которые расположены в запрещенной зоне приблизительно на 0.2 эВ ниже дна зоны проводимости [7]. Степень компенсации незначительно влияет на энергию активации.

В некоторых образцах (образцы 2-6) определены температурные зависимости подвижности носителей заряда $(\mu)$ от температуры и выяснен механизм рассеяния (рис. 2). В образцах $p$-типа проводимости (образцы 2, 3) наблюдается сложный характер $\mu(T)$, при $T<200 \mathrm{~K}$ подвижность изменяется по закону $\mu \propto T^{1.4}$. В образцах $n$-типа проводимости (образцы 4-6) в интервале температур $T<200 \mathrm{~K}$ подвижность изменяется по закону $\mu \propto T^{n}$ (где $n>2$ ), с увеличением содержания эрбия показатель степени $n$ увеличивается и в интервале температур $T>400 \mathrm{~K}$ подвижность почти не изменяется, а с изменением концентрации в образцах одновременно возникают несколько механизмов рассеяния. Различие показателей степени для электронов и дырок может быть связано с разным влиянием соответствующих акустических и оптических потенциалов деформации. Особенность изменения подвижности носителей от поляризованных центров, содержащих эрбий, при низких температурах $(T<200 \mathrm{~K})$ и механизм взаимодействия носителей заряда с фононами не полностью ясны. Для вы-

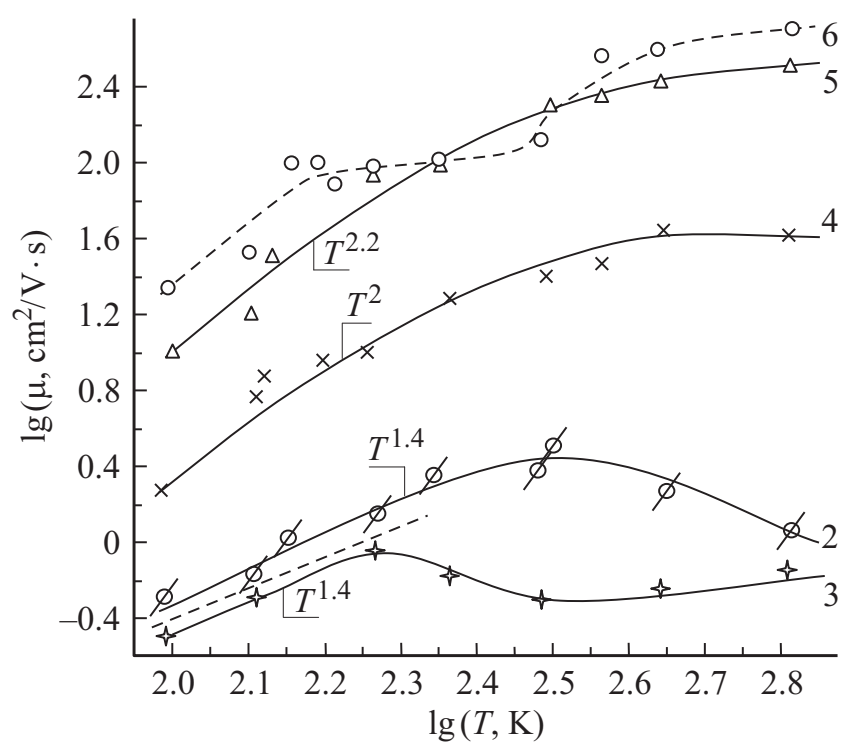

Рис. 2. Температурные зависимости подвижности носителей заряда в твердых растворах $\mathrm{Er}_{x} \mathrm{Sn}_{1-x} \mathrm{Se}$. Образцы: $2-x=0.001,3-0.0025,4-0.005,5-0.01,6-0.015$. 
Изменение некоторых кинетических параметров в твердых растворах $\operatorname{Er}_{x} \operatorname{Sn}_{1-x} \operatorname{Se}(T=90 \mathrm{~K})$

\begin{tabular}{c|c|c|c|c|c|c|c|c}
\hline $\begin{array}{c}\text { Номер } \\
\text { образца }\end{array}$ & $\begin{array}{c}p, n, \\
\mathrm{~cm}^{-3}\end{array}$ & $\begin{array}{c}\mu, \\
\mathrm{cm}^{2} / \mathrm{B} \cdot \mathrm{c}\end{array}$ & $\begin{array}{c}\alpha_{\mathrm{tot}}, \alpha_{D}, \\
10^{-6} \mathrm{~B} / \mathrm{K}\end{array}$ & $\begin{array}{c}\alpha_{T}, \\
10^{-6} \mathrm{~B} / \mathrm{K}\end{array}$ & $\begin{array}{c}\alpha(H), \\
10^{-6} \mathrm{~B} / \mathrm{K}\end{array}$ & $\begin{array}{c}\Delta \alpha, \\
10^{-6} \mathrm{~B} / \mathrm{K}\end{array}$ & $\begin{array}{c}\Delta \alpha(H), \\
10^{-6} \mathrm{~B} / \mathrm{K} \\
\text { (эксперимент) }\end{array}$ & $\begin{array}{c}\bar{\mu} \\
\end{array}$ \\
\hline 1 & $5.7 \cdot 10^{18}$ & 130 & 380 & 160 & 296 & 220 & 84 \\
3 & $3.9 \cdot 10^{16}$ & 30 & 280 & 160 & 248 & 120 & 32 \\
5 & $3.6 \cdot 10^{16}$ & 19 & -290 & 200 & -252 & -490 & 38 \\
8 & $5.4 \cdot 10^{17}$ & 45 & -152 & 200 & -131 & -352 & 21 & 0.4 \\
\end{tabular}

явления причин вышеуказанных различий между $\alpha_{\mathrm{tot}}(T)$ и $\alpha_{T}(T)$ и отклонений от линейности температурной зависимости термоэдс ниже $300 \mathrm{~K}$ проведены исследования поперечного эффекта Нернста-Эттингсгаузена (Н-Э) с использованием формулы $[9,10]$

$$
E_{y}=Q_{N}^{\perp} H \frac{\partial T}{\partial x}
$$

где $Q_{N}^{\perp}-$ коэффицент $\mathrm{H}-Э, E_{y}-$ безразмерное поперечное поле. Полученные результаты приведены на рис. 3. Из рисунка видно, что максимальное значение по абсолютной величине $Q_{N}^{\perp}$ наблюдается в интервале температур 160-240 K и с ростом температуры уменьшается. Анализируя зависимости $Q_{N}^{\perp}(T)$, наблюдаем, что в образцах $p$-типа проводимости $(1-3)$ знак эффекта отрицательный, а в образцах $n$-типа $(5,6)$ - положительный.

Различие знаков между данными, полученными из эффектов Холла и Н-Э, по-видимому, связано с возникновением в кристаллах $\mathrm{Er}_{x} \mathrm{Sn}_{1-x} \mathrm{Se}$ комплексов, в которых проявляется сильное влияние носителей заряда на последний эффект, так как возникновение комплекса при-

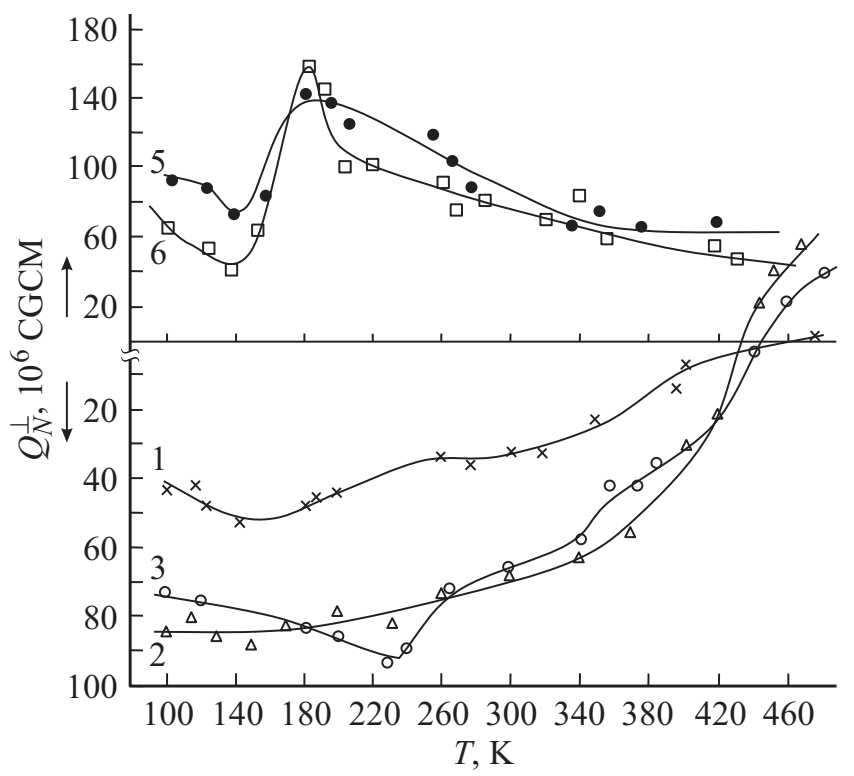

Рис. 3. Зависимости коэффициента $Q_{N}^{\perp}$ от температуры для образцов $\mathrm{Er}_{x} \mathrm{Sn}_{1-x} \mathrm{Se}: 1-x=0,2-0.001,3-0.025,5-$ $0.01,6-0.015$. водит к значительному изменению коэффицента $Q_{N}^{\perp}$ и сравнительно мало изменяет постоянную Холла. Согласно теории $[10-12]$, отрицательный знак $Q_{N}^{\perp}$ в области $T<300 \mathrm{~K}$ может быть связан с увлечением дырок фононами [13-16]. Для выявления роли фононного увлечения было выбрано два типа кристаллов: $p$-типа проводимости с концентрацией примеси $p=3.9 \cdot 10^{16} \mathrm{~cm}^{-3}$ (образец 3), $n$-типа с приблизительно такой же концентрацией примеси, $n=3.6 \cdot 10^{16} \mathrm{~cm}^{-3}$ (образец 5), в которых при $T \approx 100 \mathrm{~K}$ химический потенциал $\bar{\mu}$ равен 0.4 и 0.8 соответственно (см. таблицу), и проведены измерения продольного эффекта $\mathrm{H}-Э \Delta \alpha / \alpha(0)=[\alpha(H)-\alpha(0)] / \alpha(0)$. Согласно теории Херринга [12], вклад фононной части термоэдс можно представить в виде $\alpha_{\mathrm{ph}}=\alpha_{\text {tot }}-\alpha_{D}$, где $\alpha_{D}$ - диффузионная часть термоэдс (см. таблицу). Учитывая, что в сильных магнитных полях $H$ параметры $\alpha$ tot и $Q_{N}^{\perp}$ не зависят от механизма рассеяния и известна $\alpha_{\mathrm{ph}}(T)$ в сильных полях $(\gamma=\mu H / c \gg 1)$, определили фононную часть термоэдс в виде $[10,12]$

$$
\alpha_{\mathrm{ph}}(\infty, T)=-\left(\frac{k_{0}}{e}\right) \frac{2}{3 \pi}\left\langle A_{\mathrm{ph}}(\varepsilon)\right\rangle .
$$

Определяя в (3) $\alpha_{\text {ph }}$ при сильных полях, вычислили среднюю силу увлечения $\left\langle A_{\mathrm{ph}}(\varepsilon)\right\rangle$, позволяющую оценить константу взаимодействия. При отсутствии магнитного поля $(\gamma=0)$ термоэдс $\alpha_{\mathrm{ph}}(0)$ определялась по формуле $[10,12]$ :

$$
\alpha_{\mathrm{ph}}(0)=\frac{k_{0}}{e} \frac{\left\langle A_{\mathrm{ph}}(\varepsilon)(\tau / m)\right\rangle}{\langle\tau / m\rangle},
$$

где $\alpha(0)$ - обычная диффузионная термоэдс при $H=0$. Из (4) видно, что с ростом вырождения отношение

$$
\frac{\alpha_{\mathrm{ph}}(0)}{\alpha_{D}}=\frac{\left\langle(\tau / m) A_{\mathrm{ph}}(\varepsilon)\right\rangle /\langle\tau / m\rangle}{\langle x \tau / m\rangle /\langle\tau / m\rangle}
$$

увеличивается.

Рассчитаны $\alpha_{\mathrm{ph}}(T)$ для образцов 3 и 5 при температурах $96,180 \mathrm{~K}$ на (рис. $4, a)$ и $A_{\mathrm{ph}}(T, \varepsilon)$ в зависимости от $\gamma$ (рис. $4, b$ ) при тех же температурах, что и $\alpha_{\mathrm{ph}}(T)$. Обнаружено, что с возрастанием $\gamma A_{\mathrm{ph}}(T, \varepsilon)$ уменьшается, причем уменьшение $\alpha_{\mathrm{ph}}(\gamma)$ и $A_{\mathrm{ph}}(T, \varepsilon)$ при $96 \mathrm{~K}$ более резкое (кривая 3 ), чем при $180 \mathrm{~K}$ (кривая 1 ), что однозначно объясняется влиянием силы увлечения на $\alpha_{\mathrm{ph}}$.

Для определения среднего значения силы увлечения $\left\langle A_{\mathrm{ph}}(T, \varepsilon)\right\rangle$ была использована методика определения 


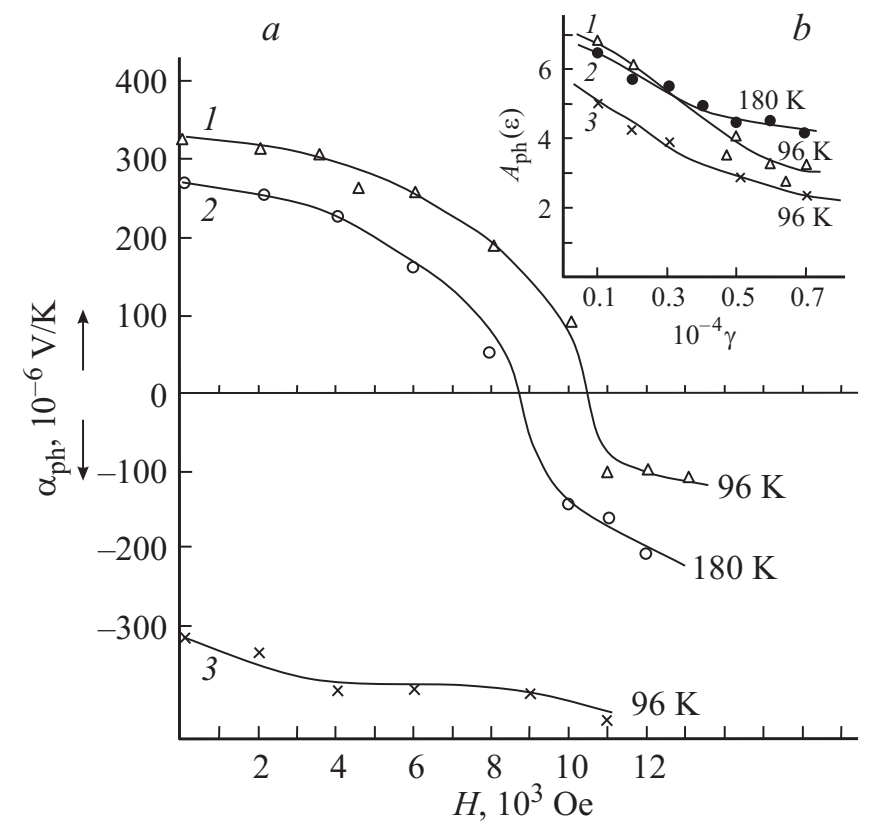

Рис. 4. Полевые зависимости $\alpha_{\mathrm{ph}}(H)(a)$ и $A_{\mathrm{ph}}(T, \gamma)(b)$ при различных температурах: 1,2 - образец $3 ; 3-$ образец 5 .

продольного термомагнитного эффекта $\mathrm{H}-Э \Delta \alpha_{\infty}(H)$, $\alpha_{\infty}=\alpha_{0}+\left|\Delta \alpha_{\infty}\right|$, согласно которой [11]

$$
\Delta \alpha_{\mathrm{ph} \infty}(\gamma)=\Delta \alpha_{\mathrm{ph}}(\gamma) \frac{1+\gamma^{2}}{\gamma^{2}} .
$$

Эксперименты показывают, что в исследованной области температур $\alpha_{\infty}$ в магнитном поле уменьшается, т. е. $\Delta \alpha_{\infty}(\gamma)$ имеет отрицательный знак. Из формул (5) определено среднее значение параметра $\left\langle A_{\mathrm{ph}}(\varepsilon)\right\rangle$ : при $T=90 \mathrm{~K}\left\langle A_{\mathrm{ph}}(\varepsilon)\right\rangle=7.4$, при $T=180 \mathrm{~K}\left\langle A_{\mathrm{ph}}(\varepsilon)\right\rangle=6.2$, при $T=300 \mathrm{~K}\left\langle A_{\mathrm{ph}}(\varepsilon)\right\rangle=6.5$. При этих же температурах вычислены отношения $\alpha_{\mathrm{ph}}(0) / \alpha_{D}(0)$, которые при температурах 96, 150, $180 \mathrm{~K}$ уменьшаются и согласуются с экспериментальными результатами. Это подтверждает вывод о том, что данное значение отношения (5) с уменьшением температуры возрастает.

Наблюдаемые эффекты влияния увлечения на термомагнитные свойства при неравновесных условиях связаны с поляризацией оптическими колебаниями решетки [14], направленными узкими каналами, которые приводят к сильному взаимодействию носителей заряда с продольными длинноволновыми оптическими фононами. Поляризованные „оптические“ колебания слабо взаимодействуют друг с другом из-за наличия ангормонического слагаемого, которое непосредственно связывает продольно и поперечно поляризованные „оптические“ колебания.

\section{4. Заключение}

Наблюдаемые отклонения $\Delta \alpha / \alpha_{0}$ от линейности в интервале температур 77-220 K связаны не только с увлечением носителей длинноволновых акустических фононов, а также в процессе участвуют поляризованные оптические фононы. Невозможно разделить колебания на продольные и поперечные в условиях поляризации. В процессе взаимодействия играют роль и поперечные фононы при неравновесных условиях. В результате в исследованном интервале температур в составе $\mathrm{Er}_{x} \mathrm{Sn}_{1-x} \mathrm{Se}$ помимо рассеяния на акустических колебаниях существуют и другие, более важные механизмы рассеяния. Так как парамагнитные ионы $\mathrm{Er}^{3+}$, создавая частично поляризацию, вызывают длинноволновые оптические фононы, доминирующие над продольными акустическими фононами; оптические колебания слабо взаимодействуют друг с другом из-за наличия ангармонического слагаемого, которое непосредственно связывает продольно и поперечно поляризованные оптические колебания.

\section{Список литературы}

[1] Х.Х. Абрикосов, Л.Е. Шелимова. Полупроводниковые материалы на основе соединения $A^{I V} B^{V I}$ (М., Наука, 1975).

[2] Л.В. Прокофьева, Ю.И. Равич, Д.А. Пшенай-Северин, П.П. Константинов, А.А. Шабалдин. ФТП, 44, 742 (2010).

[3] Ю.И. Равич, С.А. Немов. ФТП, 36, 3 (2002).

[4] В.И. Донецких, Е.Ф. Зайганов, В.П. Зломанов, С.Г. Крочтору, Е.А. Кулюхина, В.В. Соболев, В.И. Штанов. В сб.: Химчческая связь в кристаллах и их физические свойства (Изд. „Наука и техника“, Минск, 1976) т. II.

[5] А.В. Дмитриев, И.П. Звягин. УФН, 180, 821 (2010).

[6] Д.И. Гусейнов, М.И. Мургузов, Ш.С. Исмаилов. Изв. РАН Неорг. матер., 44, 542 (2008).

[7] Дж.И. Гусейнов, М.И. Мургузов, Ш.С. Исмаилов. ФТП, 47, 298 (2013).

[8] С.А. Немов, М.К. Житинская, В.И. Прошин. ФТП, 25 (1), 114 (1991).

[9] А.И. Ансельм. Введение в теорию полупроводников (М., Лань, 2008).

[10] И.М. Цидильковский. Термомагнитные явления в полупроводниках (М., Физ.-мат. лит., 1960).

[11] Б.М. Аскеров. Электронные явления переноса в полупроводниках (М., Наука, 1985).

[12] C. Herring. Phys. Rev., 95, 954 (1954).

[13] Ю.И. Равич, Б.А. Ефимова, И.А. Смирнов. Методы исследования полупроводников в применении к халькогенидам свинца PbTe, PbSe, PbS (М., Наука, 1968).

[14] Л.Э. Гуревич, Г.П. Недлик. ЖЭТФ, 40, 809 (1961).

[15] Э.Д. Кригер, А.Ф. Кравченко, Э.М. Скок. ФТП, 5, 1605 (1971).

[16] Ю.В. Гуляев, Г.Н. Шкердин. ФТТ, 21, 2695 (1979).

Редактор Л.В. Шаронова 
Thermoemf and thermomagnetic properties of $\mathrm{Er}_{x} \mathrm{Sn}_{1-x} \mathrm{Se}$ solid solutions

Y.I. Huseynov', M.I. Murguzov' ${ }^{1}$, Sh.S. Ismayilov', R.F. Mamedova ${ }^{1}$, E.M. Goiaex ${ }^{2}$

${ }^{1}$ Azerbaijan State Pedagogical University,

AZ-1000 Baku, Azerbaijan

${ }^{2}$ Azerbaijan Technical University,

AZ-1073 Baku, Azerbaijan 